\title{
Plasma-Modified Polyethylene Separator Membrane for Lithium-ion Polymer Battery
}

\author{
Jun Young Kim ${ }^{1,2}$ and Dae Young Lim $^{3}$ \\ ${ }^{1}$ Material Laboratory, Corporate RED Center, Samsung SDI Co., Ltd. South Korea \\ ${ }^{2}$ Dept. of Materials Science \& Engineering, Massachusetts Institute of Technology, USA \\ ${ }^{3}$ Fusion Textile Technology Team, Korea Institute of Industrial Technology, South Korea \\ *E-mail addresses: junykim74@hanmail.net (J.Y. Kim); zoro1967@kitech.re.kr (D.Y. Lim)
}

\begin{abstract}
This chapter describes the fabrication of a novel modified polyethylene (PE) membrane using plasma technology to create high-performance separator membrane for practical applications in rechargeable lithium-ion polymer battery. The surface of PE membrane as a separator for lithium-ion polymer battery was modified with acrylonitrile via plasmainduced coating technique. The plasma-induced acrylonitrile coated PE (PiAN-PE) membrane was characterized by X-ray photoelectron spectroscopy (XPS), scanning electron microscopy (SEM), and contact angle measurements. The electrochemical performance of lithium-ion polymer cell assembly fabricated with PiAN-PE membranes was also analyzed. The surface characterization demonstrates that the enhanced adhesion of PiAN-PE membrane resulted from the increased polar component of surface energy. The presence of PiAN induced onto the surface of PE membrane via plasma modification process plays a critical role in improving the wettability and electrolyte retention, the interfacial adhesion between the electrodes and the separator, and the cycle performance of the resulting lithium-ion polymer cell assembly. This plasma-modified PE membrane holds a great potential to be a promising polymer membrane as a high-performance and cost-effective separator for lithium-ion polymer battery. This chapter also suggests that the performance of lithium-ion polymer battery can be greatly enhanced by the plasma modification of commercial separators with proper functional materials for targeted application.
\end{abstract}

\section{Introduction}

As there is a growing demand for high-performance rechargeable batteries used in portable electronic equipments, mobile products, and communication devices, lithium-based batteries as a power source are of great scientific interests. Among many types of rechargeable batteries, lithium-ion polymer batteries hold potential to be used in industries, because they can be produced in a variety of forms and thus make it possible to fabricate readily portable batteries in required shapes for various electronic applications (Scrosati, 1993). 
A separator placed between a cathode and an anode is one of critical components in the rechargeable lithium batteries. Its primary function is to effectively transport ionic charge carriers between the two electrodes as an efficient ionic conductor as well as to prevent the electric contact between them as a good electric insulator (Linden \& Reddy, 2002; Besenhard, 1999). A separator should be chemically or electrochemically stable and have mechanical strength sufficiently enough to sustain battery-assembly processes (Besenhard, 1999; Zhang, 2007; Arora \& Zhang, 2004). In addition, a separator has a significant effect on the manufacturing process and the performance of rechargeable lithium batteries. Commercially available porous polyolefin separators have good mechanical and thermal properties and effectively prevent thermal runaway caused by electrical short-circuits or rapid overcharging. However, they do not readily absorb the electrolyte solvents with high dielectric constants such as ethylene carbonate, propylene carbonate, and $\gamma$-butyrolactone due to their hydrophobic surface with low surface energy, and have poor ability in retaining the electrolyte solutions (Wang et al., 2000; Lee et al., 2005). In addition, the solvent leakage from the interfaces between electrodes or the opposite side of current collectors often causes the deterioration of life cycle of the rechargeable lithium batteries (Croce et al., 1998). To overcome these drawbacks of conventional polyolefin separators, much research has been undertaken to develop alternative separators that are compatible with polar liquid electrolytes and stable with the electrode materials (Michot et al., 2000; Huang \& Wunder, 2001; Song et al., 2002; Saito et al., 2003).

A number of efforts have been made to achieve high-performance polyolefin separators by coating them with gel polymer electrolytes to improve the compatibility with various electrolyte solutions as well as the electrochemical properties of the lithium-ion polymer batteries (Abraham et al., 1995; Kim et al., 2001; Wang et al., 2002). Although these surfacemodified polyolefin separators exhibit good mechanical and thermal properties as well as the degree of compatibility with the electrolyte solutions, they still have several disadvantages such as complex multi-step processes and relatively expensive modification of the surface of hydrophobic polyolefin separator with adequate hydrophilic monomers to increase the surface energy enough to absorb the electrolyte solutions. Among the numerous methods of the surface modification of polyolefin separators, the radiation process is one of the most promising methods due to the rapid formation of active sites for initiating the reaction through the polymer matrix and the uniformity of polymers over the entire specimens (Tsuneda et al., 1993). In addition, plasma process is a preferred and convenient technique when considering a large scale production or commercialization of the membrane. However, studies on the surface modification of polyolefin separators using the plasma technology have rarely been investigated to date.

In this chapter, we describe the fabrication of a novel modified polyethylene (PE) membrane by coating the plasma-induced acrylonitrile (PiAN) onto the surface of PE membrane using plasma technology in order to create high-performance separator membranes for practical applications in rechargeable lithium-ion polymer batteries. An acrylonitrile was chosen as a polymeric coating material for the surface of PE membranes because of its chemical stability and ability to be easily wetted by the electrolyte solution for use in the lithium-ion polymer batteries (Choe et al., 1997; Akashi et al., 1998). Attempts to coat the PiAN on the surface of a porous PE membrane and to fabricate the plasma-induced AN coated membrane (PiAN-PE) have not been previously investigated, and the study on the characterization of PiAN-PE membranes have not yet been reported in the literature. This is the first study of possible 
realization of PiAN-PE membrane as a separator, and will help in preliminary evaluation and understanding of PiAN-PE membrane as a separator for the lithium-ion polymer battery. This study also suggests that PiAN-PE membrane via plasma treatment holds a great potential to be used as a high-performance cost-effective separator for lithium-ion polymer batteries.

\section{Fabrication of separators for lithium-ion polymer battery}

\subsection{General features}

The separator is a critical component in the lithium-ion polymer battery, and its primary function is to facilitate ionic transport between the electrodes as well as to prevent the electric contact of the electrodes. However, the presence of the separator in lithium-ion polymer battery induces electrical resistance and limited space inside the battery to satisfy the need for slimming and safety, which significantly influences the battery performance. Thus, the fabrication of high-performance separators plays an important role in controlling the overall performance of lithium-ion polymer battery, including high power or energy density, long cycle life, and excellent safety.

For many design options, the separator design requirements have been proposed by many researchers, and a number of factors influencing the battery performance must be considered in achieving high-performance separators for the battery applications. Among a wide variety of properties for the separators used in the lithium-ion battery, the following criteria should be qualified to fabricate the separators for lithium-based battery (Arora \& Zhang, 2004): (a) electronic insulator, (b) minimal electric resistance, (c) dimensional stability, (d) mechanical strength enough to allow the assembly process, (e) chemical stability against degradation by electrolyte or electrode reactants, (f) effective prevention of the migration of particles or soluble species between the electrodes, (g) good wettability on electrolyte solution, and (h) uniform thickness and pore distribution. General requirements of the separators for lithium-ion batteries are summarized in Table 1.

\begin{tabular}{l|l|l}
\hline \multicolumn{1}{c|}{ Separator parameters } & \multicolumn{1}{|c}{ Parameter values } & \multicolumn{1}{c}{ Standard } \\
\hline Thickness & $<25 \mu \mathrm{m}$ & ASTM D5947-96 \\
Electrical resistance & $<2 \Omega \mathrm{cm}^{2}$ & US 4,464,238 \\
Pore size & $<1 \mu \mathrm{m}$ & ASTM E128-99 \\
Porosity & $\sim 40 \%$ & ASTM E128-99 \\
Wettability & Completely wet in liquid electrolytes & \\
Chemical stability & Stable in the battery for long cycle life & \\
Tensile strength & $>1500 \mathrm{~kg} / \mathrm{cm}^{2}$ & ASTM D882 \\
Puncture strength & $>300 \mathrm{~g} / \mathrm{mil}^{2}$ & ASTM D3763 \\
Shrinkage & $<5 \%$ & ASTM D1204 \\
Shutdown temperature & $\sim 130^{\circ} \mathrm{C}$ & \\
\hline
\end{tabular}

Table 1. Separator requirements for lithium-ion battery

Most of microporous polymer membranes currently used in the lithium-ion polymer battery is based on polyolefin resins, including polyethylene (PE), polypropylene (PP) and their blends or multilayer forms such as PE-PP and PP-PE-PP (Higuchi et al., 1995; Sogo, 1997; Hashimoto et al., 2000; Fisher \& Wensley, 2002; Lee et al., 2004). Usually, the microporous polymer membrane as a separator for lithium-ion polymer battery can be fabricated by dry 
and wet processes, including the extrusion step to make thin films and the orientation steps to impart porosity and increase mechanical strength (Bierenbaum et al., 1974; Kim \& Lloyd, 1991). The separators made by dry process show a distinct slit-pore and straight microstructure, while those made by wet process exhibit interconnected spherical or elliptical pores (Zhang, 2007). The dry process for polymers with high crystallinity consists of the following steps (Yu \& Dwiggins, 1997; Yu, 2000; Chandavasu et al., 2004; Yu, 2005): (a) extruding step (polyolefin resins are melt-extruded into a uniaxially oriented film), (b) annealing step (to improve the size and lamellar crystallites), and (c) stretching step (i.e., annealed films are deformed along the machine direction by three sequential processes of cold stretching to create the pore structure, hot stretching to increase the size of pores, and relaxation to reduce internal stresses within the films). Consequently, the porosity of microporous membranes depends on the morphology of films, annealing conditions and stretching ratios (Arora \& Zhang, 2004; Zhang, 2007). The wet process for both crystalline and amorphous polymers is performed as follows (Kesting, 1985; Weighall, 1991; Yen et al., 1991; Chung et al., 1993; Kim et al., 1993): (a) mixing of hydrocarbon liquid and other additives with polyolefin resins and heating, (b) extrusion of the heated solution into a sheet, orientating the sheet uniaxially or biaxially, and (c) extraction of the liquid with a volatile solvent to form the microporous structure (Bierenbaum et al., 1974; Takita et al., 1991). For semi-crystalline polymers, a stretching step can be performed before/after the extraction step to achieve high porosity and a large pore size (Ihm et al., 2002).

However, commercially available polyolefin separators cannot satisfy the enhanced battery characteristics and stability accompanied by the need for sliming various devices required in the actual industrial field. Accordingly, the physical properties of polyolefin separator must be improved in order to be applied as a separator for high-performance and high-safety lithium-ion polymer battery. In addition, commercial polyolefin separators cannot be wet easily organic electrolyte solutions with high dielectric constant usually used in lithium battery, and have poor ability in conserving the electrolytes during the repeated cycling process. Further, they have a shortcoming since it causes a phenomenon of leaking organic electrolyte solutions between electrodes or separators, thereby lowering the cycle life performance of lithium-ion polymer battery. Future development of polymer membranes as separators for lithium-ion polymer batteries will be performed by balancing high performance of separators against their safety and manufacturing cost.

\subsection{Plasma treatment techniques}

Plasma treatment methods have been developed to modify polymer surfaces for enhanced adhesion, wettability, printability, dye-uptake, etc., and usually performed by modifying the surfaces on only several molecular levels, thus allowing the surface functionalization of polymers without sacrificing their appearance and bulk properties (Liston et al., 1994). Plasma is a chemical process and its chemistry determines on polymers. Among several plasma processes, cold gas plasma treatments are used in processing of them. Cold gas plasma generally has very low temperature $(300 \sim 600 \mathrm{~K})$ and particle density of $10^{10} 10^{12}$ no. $/ \mathrm{cm}^{3}$, suitable for modifying polymer materials (Kapla \& Rose, 2006). Exposing gases to sufficient electromagnetic power dissociate them, and create chemically reactive gases that modify the exposed surfaces. At the atomic level, plasma consists of ions, electrons, and various neutral species at different energy levels. One of the excited species is free radicals, which can directly react with the surface of polymers, leading to remarkable modifications 
to their chemical structures and properties. The generated ions and electrons collide with the atoms of surfaces, and transfer energy to form more radicals, ions, and atoms. The general reactions induced by means of cold gas plasma, depending on the substrate, gas chemistry, reactor design, and operating parameter, are as follows (Liston et al., 1994; Shishoo, 2007): (a) cleaning to remove organic contamination from the surfaces, (b) ablation or etching of materials form the surface of polymer to remove a weak boundary layer and increase the surface roughness and area, (c) crosslinking of near-surface molecules, to cohesively strengthen the surface layers, (d) activation by creating reactive sites, grafting of chemical moieties and functional groups to modify the chemical structures of polymer surfaces, and (e) polymerized deposition of thin polymeric films on the surface. Various gases used for plasma reactions are presented in Table 2. As oxygen gas plasma breaks the C-C bonds of polymers, volatile monomers or oligomers ablate at shorter molecules, and they are cleaned with the exhaust. After cleaning, the plasma begins ablating the top layer of polymers. Amorphous and crystalline regions will be removed at different rates, producing the surface topology with a view to increasing the mechanical adhesion. On the contrary, noble gases such as argon and helium generate free radicals on the surface and they react with adjoining radicals of molecules to form crosslinks. This process increases the strength, temperature resistance, and solvent resistance of the surface of polymers. Unlike ablation and crosslinking, certain gas or mixture of gas generate free radicals on the surface and then react with radicals of functional molecules in the plasma by covalent bonding. In particular, oxygen and tetrafluoromethane gas plasma do oxidative reaction and form polar groups such as carboxyl, hydroxyl, and hydroperoxyl on the molecules. This oxidation increases the surface energy, and enhances the hydrophilicity and wettability of polymers. If use with substances such as fluoro-monomers and acrylic-monomers, the polymerization will take place. This process provides permanent coating of thin films on the surface of polymers. In general, fluoro-monomer gas plasma provides a low surface energy and hydrophobic surfaces, while acrylic-monomer gas plasma induces permanent hydrophilicity and wettability without mixing of other gases.

\begin{tabular}{l|l}
\hline \multicolumn{1}{c|}{ Plasma reactions } & \multicolumn{1}{c}{ Gas } \\
\hline Cleaning & Oxygen \\
\hline Ablation/Etching & Argon, Helium, Oxygen \\
\hline Crosslinking & Oxygen-free noble gases such as argon and helium \\
\hline Activation & Ammonia, Argon, Helium, Methane, Nitrogen, Tetrafluoromethane \\
\hline Polymerization & $\begin{array}{l}\text { Fluoro-monomers (Hexafluoroethylene, Perfluoroallylbenzene, } \\
\text { Pentafluorostyrene, etc.) } \\
\text { Acrylic-monomers (Acrylic acid, Acrylonitrile, Alkyl acrylates, etc.) }\end{array}$ \\
\hline
\end{tabular}

Table 2. Various gases used for plasma reactions

Recently, a number of efforts have been made up to develop the performance of separators by means of plasma treatment technology, because it is very efficient techniques to modify the surface properties of polymer membranes without producing impurities or sacrificing their properties. Kubota treated PP separator film with nitrogen gas plasma to create polymeric radicals for utilizing the graft polymerization-initiating sites, followed by immersion with acrylic monomer solution and polymerization at $65^{\circ} \mathrm{C}$, and the resultant separators showed the increased ionic conductivity (Kubota, 1993). For nikel-metal secondary battery, Tsukiashi et al. modified PP non-woven fabric separators by means of 
the technique of gas plasma treatment using several gases such as oxygen, nitrogen, and argon to increase their surface hydrophilicity and they reported that the modified PP nonwoven fabric separators with the contact angle below $100^{\circ}$ showed the increased capacity retention (Tsukiashi et al., 2003). Ciszewski et al. reported the results of the plasma-induced graft polymerization of acrylic acid under UV irradiation using microporous PP membranes for nickel-cadmium (Ni-Cd) battery. (Ciszewski et al., 2006; Ciszewski et al., 2007). They modified PP membranes by argon plasma treatment to create grafting sites, followed UV irradiation to covalently-bond acrylic acid to the surface of PP, and suggested that hydrophobic surface of PP membrane changed into hydrophilic via this technique and the resulting PP membrane as a separator for Ni-Cd cells showed good mechanical properties and very low electrolytic area resistance. Choi et al., reported that the electrospun poly(vinylidene fluoride) nanofiber web treated with ethylene plasma could provide the web surface with low melting PE layer, in which polymerized-PE layer could act as a shutter by melting at elevated temperature, thus contributing to the safety of battery (Choi et al., 2004). More recently, we reported the fabrication of plasma-modified PE membrane as a separator for lithium-ion polymer battery, in which the surface of microporous PE membranes was modified with acrylonitrile using the plasma-induced coating process and the lithium-ion polymer battery cells fabricated with the modified PE separator showed the enhanced cycling life and rate performance (Kim et al., 2009).

\section{Plasma-modified polyethylene separator membrane}

Urethane acrylate (UA) and hexyl acrylate (HA) were supplied by Samsung Cheil Industry, Korea, and were used without further purification. A 2,2-azobis(2,4-dimethylvaleonitrile) (V-65®, Wako Pure Chem., Japan) was used as an initiator. The electrolyte solution of $1.3 \mathrm{M}$ lithium hexafluorophosphate $\left(\mathrm{LiPF}_{6}\right)$ dissolved in the mixture of ethylene carbonate $(\mathrm{EC})$, ethyl methyl carbonate (EMC), and dimethyl carbonate (DEC) (EC:EMC:DEC=3:2:5, by volume, battery grade) was supplied by Samsung Cheil Industry, Korea. An acrylonitrile (AN, degree of purity $>98 \%$, Aldrich) was used as received without further purification. The gel polymer electrolyte was prepared as follows: UA/HA (3:1, by weight) in the presence of an initiator were dissolved in the above electrolyte solution by stirring at room temperature to achieve better homogenization, and they were polymerized at $75^{\circ} \mathrm{C}$ for $4 \mathrm{~h}$ to produce the cross-linked gel polymer electrolytes by thermal curing. All procedures were performed in a glove box filled with argon gas.

The system consists of the reactor equipped with the inner electrodes to which an alternating voltage was applied at a frequency of $13.56 \mathrm{MHz}$, an RF power supply with an impedance matching network, and a vacuum pump. Commercial porous PE membranes (Asahi Chem., Japan) were dipped in AN solution for $5 \mathrm{~min}$, and they were moved into the reactor. Subsequently, the dipped PE membranes were placed between the electrodes in the reactor where the plasma-induced coating was initiated by the plasma generation. Prior to starting up the plasma treatment, plasma reactor was evacuated, and argon gas was introduced into the reactor at a flow rate of $400 \mathrm{sccm}$ using unit mass flow controller. Then, the vacuum pressure of the plasma reactor was maintained at a constant value of $10^{-3}$ Torr. The electrical power of the plasma was supplied by an RF power operating at $300 \mathrm{~W}$ and the treatment time was $10 \mathrm{~min}$. 
The cathode was prepared by coating the slurry consisting of $96 \%$ lithium cobalt oxide $\left(\mathrm{LiCoO}_{2}\right)$ with $2 \%$ poly(vinylidene fluoride) (PVDF) as a binder and $2 \%$ acetylene black as a conducting agent in a $N$-methyl pyrrolidone (NMP) solvent onto aluminum foils. The graphite anode was prepared by coating the slurry of $94 \%$ graphite with $6 \%$ PVDF onto copper foils. The lithium-ion polymer cells were assembled in the form of the aluminum pouch by sandwiching PE or PiAN-PE membrane between $\mathrm{LiCoO}_{2}$ cathode and graphite anode. After assembling the cell, polymer precursor solution was injected into the aluminum pouch, which then was vacuum-sealed. Polymer precursor in the cell was thermally cured in the heating oven at $75^{\circ} \mathrm{C}$ for $4 \mathrm{~h}$ to form cross-linked gel polymer electrolytes. The prepared cells were cycled once at $0.2 \mathrm{C}$ rate to improve the wetting of gel polymer electrolytes and to form stable solid electrolyte interphase (SEI) layers on the electrode surface.

XPS spectra were obtained on a VG ESCALAB 220-I system using Mg Ka X-radiation as the excitation source with a pass energy of $1253.6 \mathrm{eV}$. XPS analysis was performed under high vacuum conditions $\left(10^{-9}\right.$ Torr). All binding energies were referenced to the $C_{1 s}$ neutral carbon peak at $284.6 \mathrm{eV}$. The membrane surfaces were characterized by contact angle measurements. Water contact angle was determined by means of the sessile drop method, and the water droplet was limited to about $0.5 \mu \mathrm{l}$ to prevent gravitational distortion of its spherical profile. The surface energies of PE and PiAN-PE membranes were calculated by measuring the contact angle of two testing liquids: water and diiodomethane on the surface of the membranes at room temperature using a contact anglemeter G-1 model (ERMA Inc.). The morphology of PE and PiAN-PE membranes were observed using a JEOL JSM-6340F SEM. The peel strength was measured at room temperature by a T-peel test using an Instron 4465 testing machine employing the crosshead speed of $10 \mathrm{~mm} / \mathrm{min}$, according to the procedures in the ASTM D1876 standard. The membranes, immersed in the electrolyte solutions consisting of $1.3 \mathrm{M} \mathrm{LiPF}_{6}$ and EC:EMC:DEC (3:2:5) mixture, were sandwiched between two stainless-steel electrodes. The ionic conductivity of the membranes was obtained from the bulk resistance, which measured by AC complex impedance analysis using a Solartron 1255 frequency response analyzer over the frequency range of $100 \mathrm{~Hz}$ to 1 $\mathrm{MHz}$. The charge and discharge cycling tests of the lithium-ion polymer cell were conducted in the voltage range of $3.0 \sim 4.2 \mathrm{~V}$ at a constant current density at room temperature using a TOSCAT-300U instrument (Toyo System Co.)

\section{Effect of plasma modification on polyethylene membrane}

\subsection{Surface properties}

XPS spectra of PE and PiAN-PE membranes are shown in Figure 1. XPS analysis was performed to clarify the surface elemental composition for the membranes. XPS spectrum of PE membrane exhibited the presence of only carbon as observed at $284.6 \mathrm{eV}$, corresponding to $\mathrm{C}_{1 \mathrm{~s}}$ core level. However, PiAN-PE membrane exhibited intense and narrow peak at 532.5 $\mathrm{eV}$ and very weak intensity peak centered at $400.5 \mathrm{eV}$, corresponding to $\mathrm{O}_{1 \mathrm{~s}}$ and $\mathrm{N}_{1 \mathrm{~s}}$ core levels, respectively, as well as $\mathrm{C}_{1 \mathrm{~s}}$ core level for PE membrane, as shown in Figure 1(b). As shown in Figure 1(c), XPS spectra of $\mathrm{C}_{1 \mathrm{~s}}$ core level for PiAN-PE membrane can be decomposed into five contributions appearing at 284.6, 285.5, 286.2, 287.6, and $289.1 \mathrm{eV}$. These observed peaks were assigned to $\mathrm{C}-\mathrm{C} / \mathrm{C}-\mathrm{H}, \mathrm{C}-\mathrm{O}, \mathrm{C}-\mathrm{N}, \mathrm{C}=\mathrm{O}$, and $-\mathrm{COO}$ groups formed on the surface of PiAN-PE membrane originating from PE membrane and AN. The - 
$\mathrm{CH}$ and $-\mathrm{CH}_{2}$ (of pristine $\mathrm{PE}$ ), oxidized $(-\mathrm{C}=\mathrm{O},-\mathrm{COO},-\mathrm{COC}-$ ), and $-\mathrm{C}=\mathrm{C}-$ groups were reported to present in Ar plasma treated high density polyethylene (Svorcik et al., 2006). The percentage contributions of the $\mathrm{C}_{1 \mathrm{~s}}$ components of PiAN-PE membrane are shown in Table 3 . After the plasma-induced coating process, the contribution of the C-N groups in the XPS spectra of $\mathrm{C}_{1 \mathrm{~s}}$ core level for PiAN-PE membrane was $7.55 \%$, which was attributed to the presence of PiAN in the membrane. This result demonstrates that PiAN was effectively induced onto the surface of PE membrane via plasma treatment.
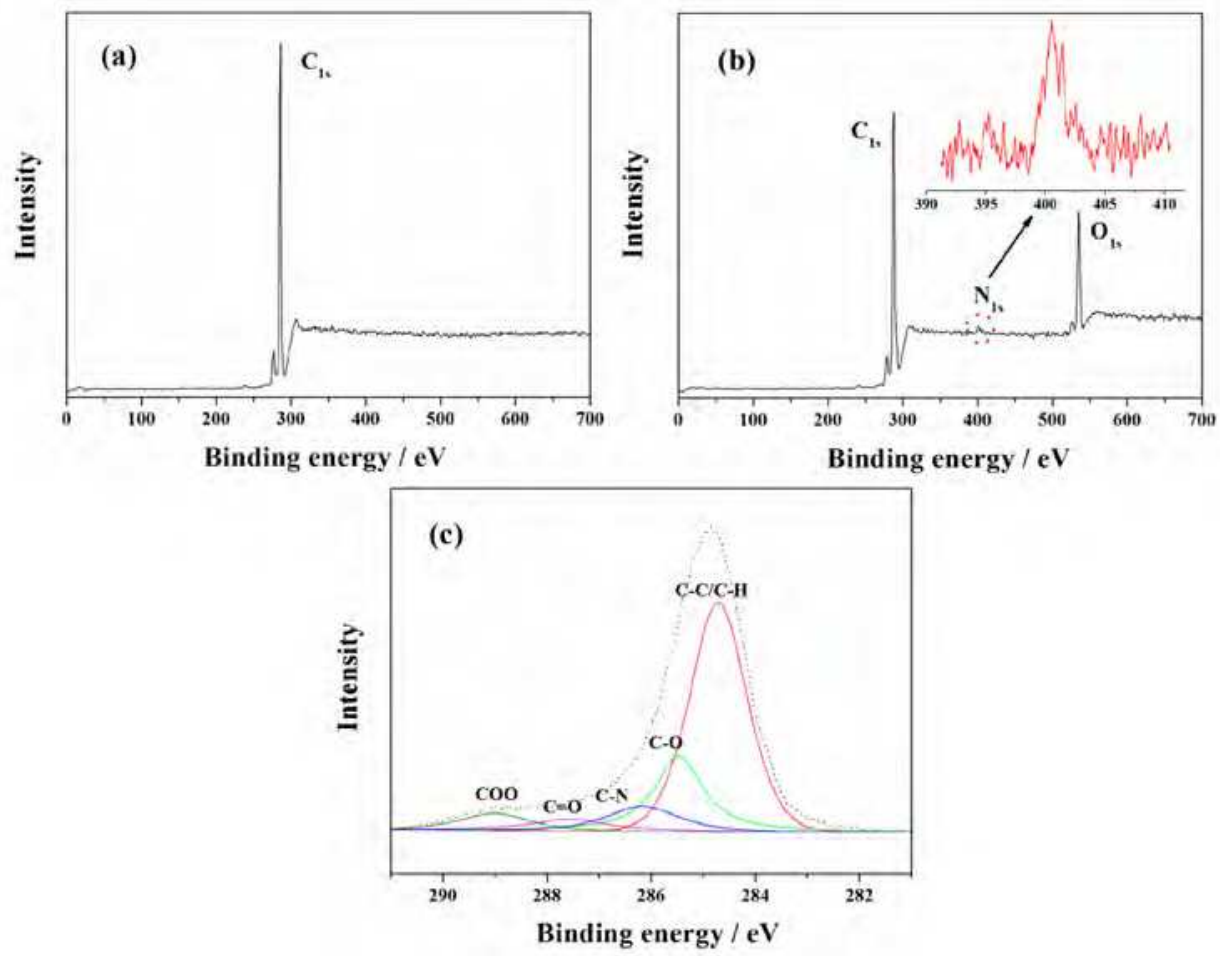

Fig. 1. XPS spectra of (a) PE and (b) PiAN-PE membranes and high resolution spectra of $C_{1 S}$ core level for PiAN-PE membranes. The inset of Fig. $1(b)$ shows $\mathrm{N}_{1 S}$ core level spectra of PiAN-PE membranes

\begin{tabular}{lccccc}
\hline Materials & \multicolumn{5}{c}{ Functional group (\%) } \\
\cline { 2 - 6 } & C-C/C- & C-O & C-N & C=O & C-O-O \\
\hline PE membrane & 98.37 & 1.16 & - & 0.26 & 0.21 \\
$\begin{array}{l}\text { PiAN-PE } \\
\text { membrane }\end{array}$ & 63.77 & 17.54 & 7.55 & 5.00 & 6.14 \\
\hline
\end{tabular}

Table 3. XPS analysis of PE and PiAN-PE membranes

The contact angle measurements of PE and PiAN-PE membranes were conducted to clarify the effect of PiAN on the surface property of the membrane. For the lithium-ion polymer cell 
assembly, the wettability of the separator used in the non-aqueous electrolytes plays a critical role in the cell performance because the separator with good wettability can effectively retain the electrolyte solutions and facilitates the electrolytes to diffuse well into the cell assembly (Arora \& Zhang, 2004; Zhang, 2007). As shown in Figure 2, the contact angle of PiAN-PE membranes significantly decreased, indicating significant change of the surface property of PE membranes. The contact angle of PiAN-PE membrane was much smaller than that of PE membrane, implying that PiAN-PE membrane has better wettability as compared to PE membrane. This result demonstrates that the surface energy of PiAN-PE membrane increased by the presence of PiAN on the surface of the membrane effectively induced via plasma treatment. Therefore, it is expected that the presence of PiAN on the surface of the membranes makes it possible for them to have high surface energy to be wetted more sufficiently in the electrolyte solution as compared to PE membrane.

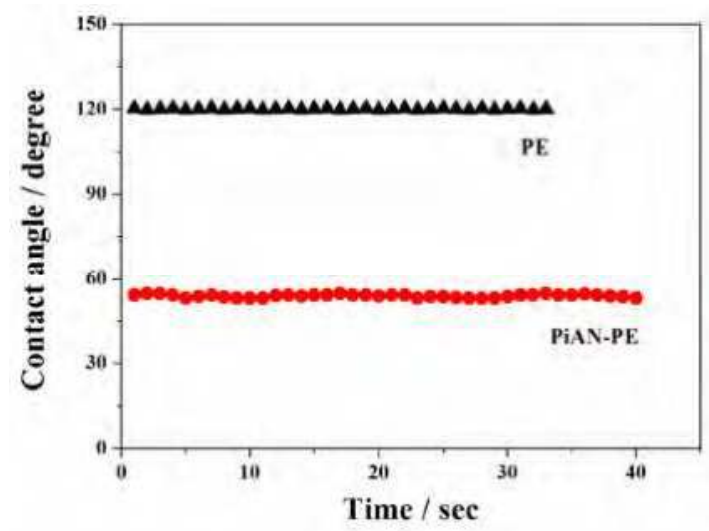

Fig. 2. Contact angles of PE and PiAN-PE membranes

The surface energy and its polar component of the PE and the PiAN-PE membranes can be estimated from the Qwens-Wendt equation modified by Fowkes and Kinloch (Owens \& Wendt, 1969; Konloch, 1987; Novak \& Chodak, 2006; Novak et al., 2008):

$$
\begin{gathered}
(1+\cos \theta) \gamma_{L V}=2 \sqrt{\left(\gamma_{L V}^{d} \cdot \gamma_{S}^{d}\right)}+2 \sqrt{\left(\gamma_{L V}^{p} \cdot \gamma_{S}^{p}\right)} \\
\gamma_{S}=\gamma_{S}^{d}+\gamma_{S}^{p}
\end{gathered}
$$

where $\theta$ is the observed contact angle; $\gamma_{\mathrm{LV}}$ and $\gamma_{\mathrm{S}}$ are the surface free energy of testing liquid and a polymer, respectively, and the superscript $d$ and $p$ refer to the dispersive and polar components of surface energy, respectively. The preferred values of the surface energy and its components for two testing liquids used are as follows: $\gamma_{\mathrm{L}}=72.8, \gamma_{\mathrm{L}}{ }^{\mathrm{d}}=21.8$, and $\gamma_{\mathrm{L}} \mathrm{p}=51.0$ $\mathrm{mJ} / \mathrm{m}^{2}$ for water and $\gamma_{\mathrm{L}}=50.8, \gamma_{\mathrm{L}}^{\mathrm{d}}=50.4$, and $\gamma_{\mathrm{L}} \mathrm{p}=0.4 \mathrm{~mJ} / \mathrm{m}^{2}$ for diiodomethane $(\mathrm{Wu}, 1982)$. The results for the surface energy of PE and PiAN-PE membranes are presented in Table 4. The PiAN-PE membrane exhibited higher values of $\gamma_{S}, \gamma_{S} p$, and $X_{p}$ than those of PE membrane. This result indicated that the presence of the PiAN in the membrane effectively 
induced via plasma-induced coating process increased the fraction of polar component in the surface energy of PiAN-PE membrane, resulting in the enhanced polarity and higher surface energy of PiAN-PE membrane. It should be noted that the increased polar component in the surface energy of PiAN-PE membrane can favor the enhancement of the interfacial adhesion between the membrane and the electrodes, thus contributing to the improvement in the cycle performance of the lithium-ion polymer cell assembly.

\begin{tabular}{lcccc}
\hline Materials & $\gamma_{\mathrm{s}}$ & $\gamma_{\mathrm{s}}{ }^{\mathrm{d}}$ & $\gamma_{\mathrm{s}} \mathrm{p}$ & $\mathrm{X}_{\mathrm{p}}{ }^{\mathrm{a}}$ \\
\hline PE membrane & 30.3 & 28.9 & 1.4 & 0.05 \\
PiAN-PE membrane & 56.6 & 40.8 & 15.8 & 0.28 \\
\hline
\end{tabular}

Table 4 . The surface energy and polarity of PE and PiAN-PE membranes [a The polarity, $X_{p}$ $\left.=\gamma_{\mathrm{p}} / \gamma\right]$

\subsection{Morphology}

SEM images of the surfaces for PE and PiAN-PE membranes are shown in Figure 3. The PE membranes had highly porous structures with uniform pore sizes of approximately $200 \mathrm{~nm}$. According to the supplier's specification, PE membranes exhibit a pore structure with the thickness of $23 \mu \mathrm{m}$ and the porosity of $\sim 40 \%$. After the plasma-induced coating process, PiAN-PE membranes exhibited rough surfaces and expanded pore structure relative to the PE membranes. The surfaces of PiAN-PE membrane appeared to be porous structures with some dense or coated layers. This result suggests that some pores or surfaces for PiAN-PE membranes may be partially covered by the PiAN. In addition, the PiAN covered in both top and bottom sides of the surface of PiAN-PE membrane can be also observed, implying that the PiAN were introduced simultaneously into both sides of the surface of PiAN-PE membrane via this plasma-induced coating process. In general, the presence of the pores on the membrane can lead to the efficient uptake of the electrolyte solution. Although both membranes were easily wetted in a few seconds in contact with the electrolyte solution, PiAN-PE membranes exhibited better wettability than PE membrane. This result was in good agreement with much lower contact angle and higher surface energy of PiAN-PE membranes as compared to the PE membranes.
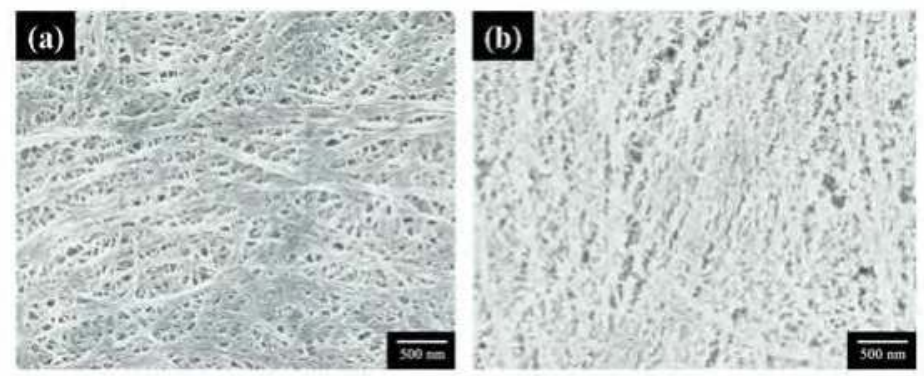

Fig. 3. SEM micrographs of the surfaces for (a) PE and (b) PiAN-PE membranes. 


\section{Plasma-modified polyethylene separator for lithium-ion polymer battery}

\section{1 lonic conductivity and adhesion}

For the liquid electrolyte-based system, the ionic conduction may be mainly provided by the electrolyte solution, and the variation of the ionic conductivity is related to the polymer morphology and microstructure of the membrane for the retention of the electrolyte solution (Linden \& Reddy, 2002). The ionic conductivities of PE and PiAN-PE membrane at room temperature were estimated to be $0.8 \times 10^{-3}$ and $1.4 \times 10^{-3} \mathrm{~S} / \mathrm{cm}$, respectively. In general, non-polar polyethylene separators exhibited poor wettability and electrolyte retention with the electrolyte solutions containing polar solvents, due to their inherent hydrophobic properties. For PE membrane, it was difficult to be completely wetted by organic solvents with high dielectric constant because of its hydrophobic surface with low surface energy, leading to lower ionic conductivity. However, PiAN-PE membrane exhibited better electrolyte retention as compared to PE membrane due to its good compatibility between the PiAN and the carbonate-based electrolyte solution, resulting from the fact that the electrolyte solution was well retained in the porous membrane by polymer-solvent interactions (Kim, et al., 2002) with the presence of the PiAN induced onto the surface of PiAN-PE membrane. Therefore, PiAN-PE membrane exhibited high ionic conductivity due to the improved wettability and electrolyte retention, resulting from the presence of the PiAN induced onto the surface of PiAN-PE membrane by the plasma treatment.

The peel testing of PE and PiAN-PE membranes was performed to clarify the effect of the PiAN induced onto the surface of PE membrane via plasma-induced coating process on the interfacial adhesion between the electrodes and the membrane. As shown in Table 5, the average peel strength of the test cell based on PiAN-PE membrane was increased to 22.6 $\mathrm{N} / \mathrm{m}$ by up to $18 \%$ compared to the reference cell with PE membrane. This result suggested that the adhesion between the separator and the electrodes was improved by the PiAN effectively induced onto the surface of the membrane via plasma-induced coating process

\begin{tabular}{lcc}
\hline Materials & Average load $(\mathrm{N})$ & Peel strength $(\mathrm{N} / \mathrm{m})$ \\
\hline PE membrane & 0.44 & 19.1 \\
PiAN-PE membrane & 0.52 & 22.6 \\
\hline
\end{tabular}

Table 5. The peel testing of PE and PiAN-PE membranes

\subsection{Cycle performance of lithium-ion polymer battery}

The lithium-ion polymer cells fabricated with PE or PiAN-PE membranes were subjected to charge-discharge tests after preconditioning with cut-off voltages of $4.2 \mathrm{~V}$ for the upper limit and 3.0 V for the lower limit at $1 \mathrm{C}$ rate. The charge-discharge profiles of the fabricated lithium-ion polymer cells with cycle number are shown in Figure 4 . It can be seen that the voltage drop in passing from charge to discharge was small, indicating the lower resistance of the lithium-ion polymer cell. As cycle number increased, the voltage and capacity decreased, which was attributed to the high polarization resulting from the increased internal resistance of the cell and the decrease in the diffusivity of the lithium ion in the electrode (Avora \& Zhang, 2004; Zhang, 2007). As shown in Figure 4, the discharge capacity of the lithium-ion polymer cell fabricated with PiAN-PE membrane was higher than that of the cell with PE membrane. The plasma-induced PiAN coating process can enhance the interfacial adhesion between the electrodes and the separator, which were interfacial 
resistance of the cell, resulting in higher cycle performance of the lithium-ion polymer cell fabricated with PiAN-PE membrane.
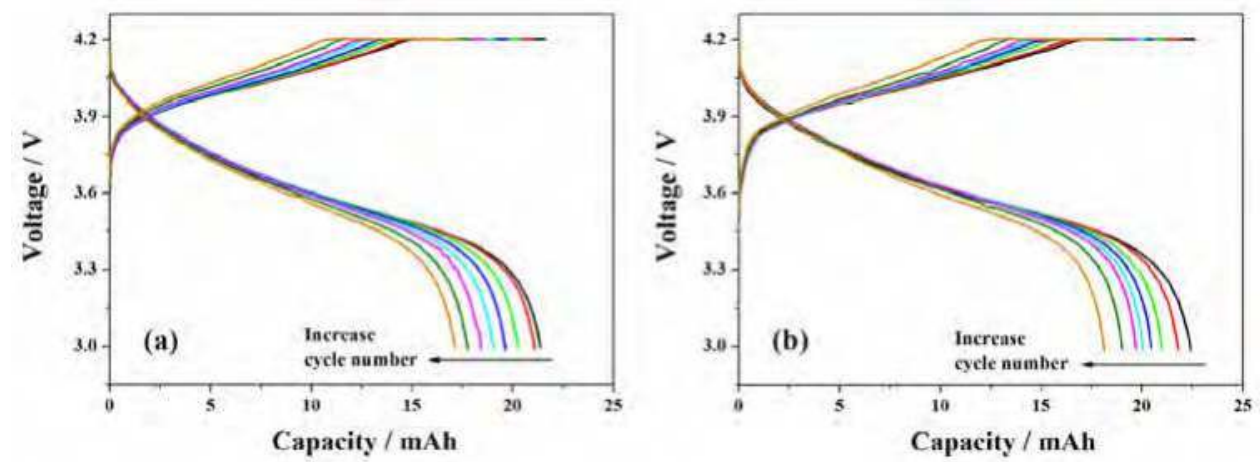

Fig. 4. Charge-discharge profiles for the lithium-ion polymer cells fabricated with (a) PE and (b) PiAN-PE membranes with increasing cycle number.

The variations of the coulombic efficiency of the lithium-ion polymer cell with cycle number are shown in Figure 5(a). In general, the coulombic efficiency can be defined as the ratio of the discharge capacity to charge capacity. The relatively low coulombic efficiency of both membranes during the initial cycling was attributed to the formation of SEI layers on the surface of the graphite electrode (Morzilli et al., 1987; Aurbach et al., 1994; Zhang et al., 2001), providing the good stability to the graphite anode toward the electrolyte reduction during lithium intercalation-deintercalation. The coulombic efficiency of the cell tended to increase with increasing cycle number, and gradually approached to unit value $(>99.5 \%)$. The discharge capacities of the lithium-ion polymer cells fabricated with PE or PiAN-PE membranes with cycle number are shown in Figure 5(b). The discharge capacity of the cell was slowly decreased with increasing cycle number. The decrease in the discharge capacity may be attributed to the deterioration of the interfacial contact of the electrodes and the physical changes in the active materials for the electrodes (Avora \& Zhang, 2004; Zhang, 2007), leading to the gradual increase in the internal resistance of the cell during the repeated charge-discharge cycling. After 96 th cycles, the lithium-ion polymer cell fabricated with PiAN-PE membrane retained $80.3 \%$ of the initial discharge capacity, while the cell with PE membrane showed $76.4 \%$ of that. Higher discharge capacity of the lithium-ion polymer cell fabricated with PiAN-PE membrane may be attributed to the enhanced wettability and the reduced interfacial resistance (Kim et al., 2004). This result suggests that the PiAN effectively induced on the surface of the membranes play a critical role in determining the cycle performance of the lithium-ion polymer cell and that stable cycle performance can be obtained by modifying the surface of PE membrane with the plasma-induced coating process. 

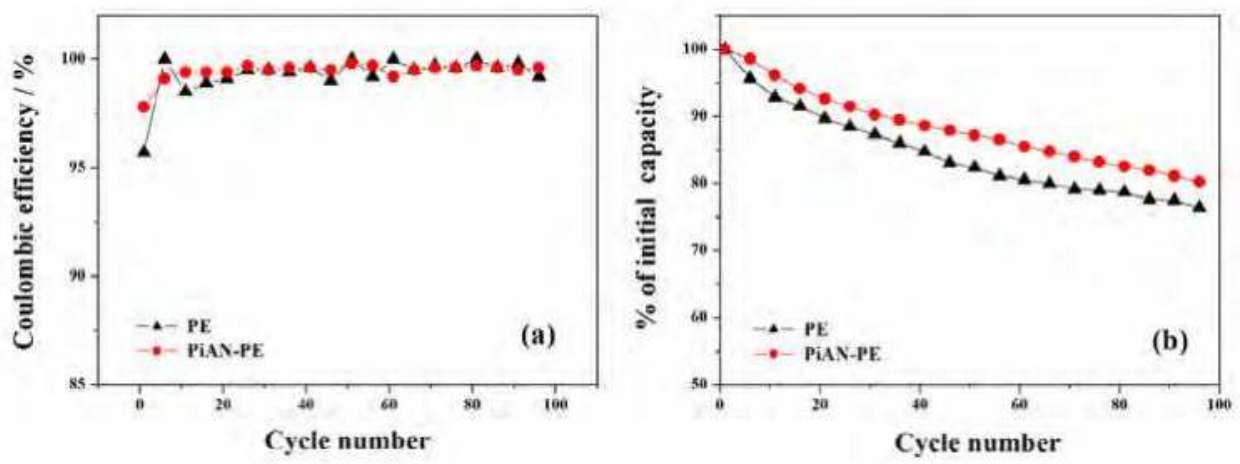

Fig. 5. (a) Coulombic efficiency and (b) discharge capacity of the lithium-ion polymer cells fabricated with PE or PiAN-PE membrane as a function of cycle number

The rate capabilities of the lithium-ion polymer cells fabricated with PE or PiAN-PE membranes with cycle number are shown in Figure 6. The lithium-ion polymer cells fabricated with PE or PiAN-PE membranes exhibited the capacity retentions of 90.3 and $93.1 \%$, respectively, at $0.5 \mathrm{C}$ rate, and then decreased clearly with increasing the current rate. The lithium-ion polymer cells fabricated with PiAN-PE membranes exhibited higher capacity retention than in the case of PE membrane. As compared to PE membrane, better rate capability of the cell fabricated with PiAN-PE membrane may be attributed to the enhanced interfacial adhesion between the electrodes and the separator as well as better wettability and the electrolyte retention. This result demonstrates that good cycle performance of the lithium-ion polymer cells can be obtained by using a novel modified PiAN-PE membrane with simple plasma treatment, suggesting that PiAN-PE membrane is expected to be a promising polymer membrane as a high-performance and cost-effective separator for rechargeable lithium-ion polymer batteries.

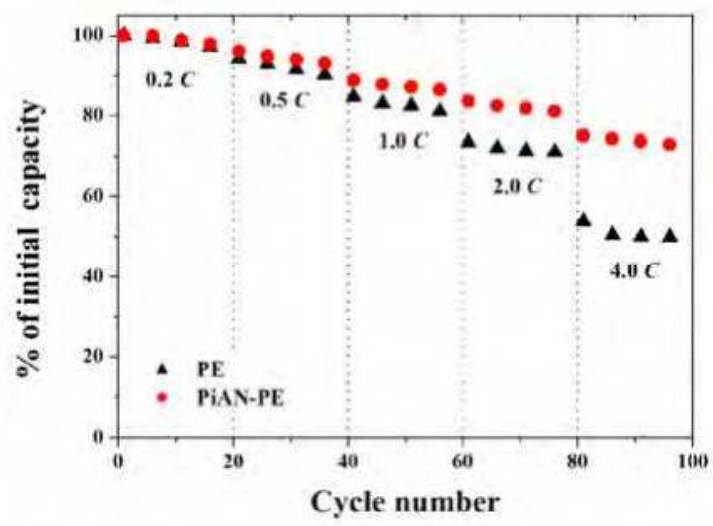

Fig. 6. Rate capability of the lithium-ion polymer cells fabricated with PE or PiAN-PE membrane as a function of cycle number 


\section{Summary}

The separator is a critical component in the lithium-ion polymer batteries, and its primary function is to facilitate ionic transport between the electrodes as well as to prevent the electric contact of the electrodes. This chapter describes the fabrication of a novel modified polyethylene membrane via plasma-induced coating process to create high performance and cost-effective separator membranes for practical applications in rechargeable lithium-ion polymer battery. The enhanced interfacial adhesion and cycle performance of the lithiumion polymer cell fabricated with modified polyethylene membrane by plasma-induced coating process were observed. The surface modification of polyethylene membrane by the plasma-induced coating process played a critical role in determining the performance of the resultant lithium-ion polymer cell assembly. The plasma-modified polyethylene membrane exhibited the increased ionic conductivity, the good wettability, and the enhanced interfacial adhesion between the electrodes and the separators. Consequently, the lithium-ion polymer cell fabricated with the plasma-modified polyethylene membrane exhibited better cycle performance as compared to the unmodified polyethylene membrane. This chapter suggests that the performance of the lithium-ion polymer battery can be greatly enhanced by the plasma modification of the commercial separator with proper materials for targeted application. Future development of microporous polymer membranes as separators for lithium-ion polymer batteries will be performed by balancing high performance of separators against their safety and manufacturing cost.

\section{References}

Abraham, K. M.; Alamgir, M. \& Hoffman, D. K. (1995). Polymer Electrolytes Reinforced by Celgard ${ }^{\circledR}$ Membranes. J. Electrochem. Soc., 142, 683-687.

Akashi, H.; Tanaka, K. \& Sekai, K. (1998). An Ionic Conductivity and Spectroscopic Study of Ionic Transport Mechanism in Fire-Retardant Polyacrylonitrile-Based Gel Electrolytes for Li Polymer Batteries. J. Electrochem. Soc., 145, 881-887.

Arora, P. \& Zhang, Z. (2004). Battery Separators. Chem. Rev., 104, 4419-4462.

Aurbach, D.; Weissman, I.; Zaban, A. \& Chusid, O. (1994). Correlation Between Surface Chemistry, Morphology, Cycling Efficiency and Interfacial Properties of Li Electrodes in Solutions Containing Different Li Salts. Electrochim. Acta, 39, 51-71.

Besenhard, J. O. (1999). Handbook of Battery Materials, Wiley-VCH, Weinheim

Bierenbaum, H. S.; Isaacson, R. B.; Druin, M. L. \& Plovan, S. G. (1974). Microporous Polymeric Films. Ind. Eng. Chem. Prod. Res. Dev., 13, 2-9.

Chandavasu, C.; Xanthos, M.; Sirkar, K. K. \& Gogos, C. (2004). Preparation of Microporous Films from, Immiscible Blends via Melt Processing and Stretching. US Patent $6,824,680$.

Choi, S. S.; Lee, Y. S.; Joo, C. W.; Lee, S. G.; Park, J. K. \& Han, K. S. (2004). Electrospun PVDF Nanofiber Web As Polymer Electrolyte or Separator. Electrochim. Acta, 50, 339-343.

Choe, H. S.; Carroll, B. G.; Pasquariello, D. M. \& Abraham, K. M. (1997). Characterization of Some Polyacrylonitrile-Based Electrolytes. Chem. Mater., 9, 369-379.

Chung, T. S.; Foley, P.; Kafchinski, E. R. (1993). Development of Poly(ethylene tetrafluoroethylene) Microporous Film for Advanced Batteries. J. Mater. Sci.-Mater. Electron., 4, 259-266. 
Ciszewski, A.; Gancarz, I.; Kunicki, J. \& Marek, B. (2006). Plasma-modified Polypropylene Membranes As Separators in High Power Alkaline Batteries. Surf. Coat. Technol., 201, 3676-3684.

Ciszewski, A.; Kunicki, J. \& Gancarz, I. (2007). Usefulness of Microporous Hydrophobic Polypropylene Membranes After plasma-induced Graft Polymerization of Acrylic Acid for High Power Nickel-Cadmium Batteries. Electrochim. Acta, 52, 5207-5212.

Croce, F.; Appetecchi, G. B.; L. Persi, L. \& Scrosati, B. (1998). Nanocomposite Polymer Electrolytes for Lithium Batteries. Nature, 394, 456-458.

Fisher, H. M. \& Wensley, C. G. (2002). Polypropylene Microporous Membrane for Battery Separator. US Patent 6,368,742.

Hashimoto, A.; Yagi, K. \& Mantoku, H. (2000). Porous Film of High Molecular Weight Polyolefin and Process for Producing Same. US Patent 6,048,607.

Higuchi, H.; Matsushita, K.; Ezoe, M. \& Shinomura, T. (1995). Porous Film, Process for Producing the Same and Use of the Same. US Patent 5,385,777.

Huang, H. \& Wunder, S. L. (2001). Ionic Conductivity of Microporous PDVF-HFP/PS Polymer Blends. J. Electrochem. Soc., 148, A279-A283.

Ihm, D. W.; Noh, J. G. \& Kim, J. Y. (2002). Effect of Polymer Blending and Drawing Conditions on Properties of Polyethylene Separator Prepared for Li-ion Secondary Battery. J. Power Sources, 109, 388-393.

Kaplan, S. L. \& Rose, P. W. (2006). Plasma Surface Treatment, In: Coatings Technology Handbook, Tracton, A. A., (Ed.), CRC Press.

Kesting, R. E. (1985). Synthetic Polymeric Membranes, John Wiley \& Sons Inc., New York.

Kim, D. W.; Ko, J. M.; Chun, J. H.; Kim, S. H. \& Park, J. K. (2001). Electrochemical Performances of Lithium-Ion Cells Prepared with Polyethylene oxide-Coated Separators. Electrochem. Commun., 3, 535-538.

Kim, D. W.; Noh, K. A.; Min, H. S.; Kang, D. W. \& Sun, Y. K. (2002). Porous Polyacrylonitrile Membrane for Lithium-Ion Cells. Electrochem. Solid-State Lett., 5, A63-A66.

Kim, J. J.; Kim, S. S.; Hwang, J. R. \& Suh, S. B. (1993). Process for the Preparation of Porous Polyolefin Separation Membranes via Thermally-Induced Phase Separation. US Patent 5,250,240.

Kim, J. Y.; Kim, S. K.; Lee, S. J.; Lee, S. Y.; Lee, H. M. \& Ahn, S. (2004). Preparation of Microporous Gel Polymer for Lithium Ion Polymer Battery. Electrochim. Acta, 50, 363-366.

Kim, J. Y.; Lee, Y. \& Lim, D. Y. (2009). Plasma-modified Polyethylene Membrane As a Separator For Lithium-Ion Polymer Battery. Electrochim. Acta, 54, 3714-3719.

Kim, S. S. \& Lloyd, D. R. (1991). Microporous Membrane Formation via Thermally-Induced Phase Separation. III. Effect of Thermodynamic Interactions on the Structure of Isotactic Polypropylene Membranes. J. Membr. Sci., 64, 13-29.

Konloch, A. J. (1987). Adhesion and Adhesives: Science and Technology, Springer

Kubota, T. (1993). Secondary Battery with Graft-polymerized Separator. US Patent 5,270,137.

Lee, Y. M.; Kim, J. W.; Choi, N. S.; Lee, J. A.; Seol, W. H. \& Park, J. K. (2005). Novel Separator Based on PVdF and PE Non-woven Matrix for Rechargeable Lithium Batteries. J. Power Sources, 139, 235-241.

Lee, S. Y.; Ahn, B. I.; Im, S. G.; Park, S. Y.; Song, H. S. \& Kyung, Y. J. (2004). High Crystalline Polypropylene Microporous Membrane, Multi-Component Microporous Membrane and Methods for Preparing the Same. US Patent 6,830,849. 
Linden, D. \& Reddy, T. B. (2002). Handbook of Batteries, McGraw-Hill, New York

Liston, E. M.; Martinu, L. \& Werheimer, M. R. (1994). Plasma Surface Modification of Polymers for Improved Adhesion: A Critical Review, In: Plasma Surface Modification of Polymers: Relevance to Adhesion, Strobel, M.; Lyons, C. S. \& Mittal, K. L., (Eds.), VSP, Netherlands.

Michot, T.; Nishimoto, A. \& Watanabe, M. (2000). Electrochemical Properties of Polymer Gel Electrolytes Based on Poly(vinylidene fluoride) Copolymer and Homopolymer. Electrochim. Acta, 45, 1347-1360.

Morzilli, S.; Bonini, F. \& Scrosati, B. (1987). Characteristics of the Lithium Electrode in Organic and Polymeric Electrolytes. Electrochim. Acta, 32, 961-964

Novak, I.; Pollak, V. \& Chodak, I. (2006). Study of Surface Properties of Polyolefins Modified by Corona Discharge Plasma. Plasma Process. Polym., 3, 355-364.

Novak, I.; Elyashevich, G. K.; Chodak, I.; Olifirenko, A. S.; Steviar, M.; Spirkova, M.; Saprykina, N.; Vlasova, E. \& Kleinova, A. (2008). Polymer Matrix of Polyethylene Porous Films Functionalized by Electrical Discharge Plasma. Eur. Polym. J., 44, 2702-2707.

Owens, D. K. \& Wendt, R. C. (1969). Estimation of the Surface Free Energy of Polymers. J. Appl. Polym. Sci., 13, 1741-1747.

Saito, Y.; Stephan, A. M. \& Kataoka, H. (2003). Ion Conduction Mechanisms of Lithium Gel Polymer Electrolytes Investigated by the Conductivity and Diffusion Coefficient. Solid State Ionics, 160, 149-153.

Scrosati, B. (1993). Applications of Electroactive Polymer, Chapman \& Hall, London

Shishoo, R. (2007). Plasma Technologies for Textiles, Woodhead Publishing Limited, England.

Sogo, H. (1997). Separator for a Battery Using an Organic Electrolytic Solution and Method for Preparing the Same. US Patent 5,641,565.

Song, J. Y.; Cheng, C. L.; Wang, Y. Y. \& Wan, C. C. (2002). Microstructure of Poly(vinylidene fluoride)-Based Polymer Electrolyte and Its Effect on Transport Properties. J. Electrochem. Soc., 149, A1230-A1236.

Svorcik, V.; Kolarova, K.; Slepicka, P.; Mackova, A.; Novotna, M. \& Hnatowicz, V. (2006). Modification of Surface Properties of High and Low Density Polyethylene by Ar Plasma Discharge. Polym. Degrad. Stab., 91, 1219-1225.

Takita, K.; Kono, K.; Takashima, T. \& Okamoto, K. (1991). Microporous Polyolefine Membrane and Method of Producing Same. US Patent, 5,051,183.

Tsuneda, S.; Saito, K.; Furusaki, S.; Sugo, T. \& Makuuchi, K. (1993). Simple Introduction of Sulfonic Acid Group onto Polyethylene by Radiation-Induced Cografting of Sodium Styrenesulfonate with Hydrophillic Monomers. Ind. Eng. Chem. Res., 32, 1464-1470.

Tsukiashi, M.; Teraoka, H.; Hata, K. \& Tajima, M. (2003). Battery Separator and Manufacturing Method Thereof, and Alkali Secondary Battery Having the Separator Incorporated Therein. US Patent 6,723,809.

Wang, H. P.; Huang, H. \& Wunder, S. L. (2000). Novel Microporous Poly(vinylidene Fluoride) Blend Electrolytes for Lithium-Ion Batteries. J. Electrochem. Soc., 147, 28532861.

Wang, Y.; Travas-Sejdic, J. \& Steiner, R. (2002). Polymer Gel Electrolyte Supported with Microporous Polyolefin Membranes for Lithium Ion Polymer Battery. Solid State Ionics, 148, 443-449. 
Weighall, M. J. (1991). Recent Advances in Polyethylene Separator Technology. J. Power Sources, 34, 257-268.

Wu, S. (1982). Polymer Interface and Adhesion, Marcel Dekker, New York

Yen, Y. L.; Lopatin, G.; Malarkey, H. \& Soane, D. (1991). Process for Producing Fluorocarbon Membranes and Membrane Product. US Patent 5,032,274.

Yu, W. C. \& Dwiggins, C. F. (1997). Methods of Making Cross-Ply Microporous Membrane Battery Separator, and the Battery Separators Made Thereby. US Patent 5,667,911.

Yu, W. C. (2005). Continuous Methods of Making Microporous Battery Separators. US Patent 6,878,226.

Yu, T. H. (2000). Trilayer Battery Separator. US Patent 6,080,507.

Zhang, S. S.; Ding, M. S.; Xu, K.; Allen, J. \& Jow, T. R. (2001). Understanding of Solid Electrolyte Interface Film Formation on Graphite Electrodes. Electrochem. Solid-State Lett., 4, A206-A208.

Zhang, S. S. (2007). A Review on the Separators of Liquid Electrolyte Li-Ion Batteries. J. Power Sources, 164, 351-364. 


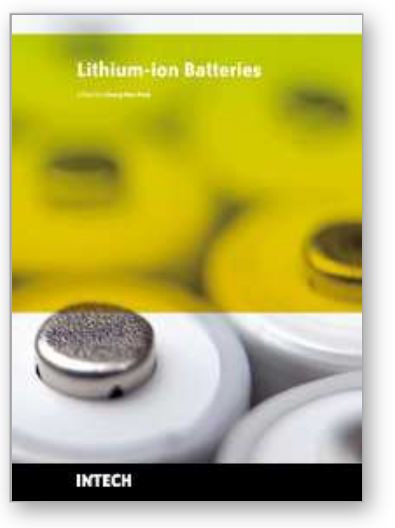

\author{
Lithium-ion Batteries \\ Edited by Chong Rae Park
}

ISBN 978-953-307-058-2

Hard cover, 132 pages

Publisher InTech

Published online 01, April, 2010

Published in print edition April, 2010

There have been numerous excellent books on LIBs based on various different viewpoints. But, there is little book available on the state of the art and future of next generation LIBs, particularly eventually for EVs and HEVs. This book is therefore planned to show the readers where we are standing on and where our R\&Ds are directing at as much as possible. This does not mean that this book is only for the experts in this field. On the contrary this book is expected to be a good textbook for undergraduates and postgraduates who get interested in this field and hence need general overviews on the LIBs, especially for heavy duty applications including EVs or HEVs.

\title{
How to reference
}

In order to correctly reference this scholarly work, feel free to copy and paste the following:

Jun Young Kim and Dae Young Lim (2010). Plasma-Modified Polyethylene Separator Membrane for Lithiumion Polymer Battery, Lithium-ion Batteries, Chong Rae Park (Ed.), ISBN: 978-953-307-058-2, InTech, Available from: http://www.intechopen.com/books/lithium-ion-batteries/plasma-modified-polyethylene-separatormembrane-for-lithium-ion-polymer-battery

\section{INTECH}

open science | open minds

\section{InTech Europe}

University Campus STeP Ri

Slavka Krautzeka 83/A

51000 Rijeka, Croatia

Phone: +385 (51) 770447

Fax: +385 (51) 686166

www.intechopen.com

\section{InTech China}

Unit 405, Office Block, Hotel Equatorial Shanghai

No.65, Yan An Road (West), Shanghai, 200040, China

中国上海市延安西路65号上海国际贵都大饭店办公楼 405 单元

Phone: +86-21-62489820

Fax: $+86-21-62489821$ 
(C) 2010 The Author(s). Licensee IntechOpen. This chapter is distributed under the terms of the Creative Commons Attribution-NonCommercialShareAlike-3.0 License, which permits use, distribution and reproduction for non-commercial purposes, provided the original is properly cited and derivative works building on this content are distributed under the same license. 